\title{
ANALYSE CONVERSATIONNELLE ET DIDACTIQUE DE LA TRADUCTION
}

\begin{abstract}
A bstract. Stefanink Bernd, Analyse conversationnelle et didactique de la traduction [Conversational Analysis and the Didactics of Translation]. Studia Romanica Posnaniensia, Adam Mickiewicz University Press, Poznań, vol. XXV/XXVI: 2000, pp. 283-298, ISBN 83-232-0965-0, ISSN 0137-2475.

The analysis of conversations held by two or more students negotiating a joint version of a translation has an important didactic value. It enables the teacher to work on the translational behavior of the students instead of merely limiting her/his activity to the correction of diverse grammar and semantic mistakes. The confrontation with their own translation procedures heightens the students' awareness of what it really means to translate. In many cases it leads to a catharsis which in turn motivates the students to study the different factors involved in the translation process. On a larger scale, conversational analysis provides the teacher with a sound basis on which to structure a translation course and organize a syllabus.
\end{abstract}

Remarques liminaires: Situation didactique à l'heure actuelle dans les cours de traduction à l'université. Les méthodes introspectives au secours d'une didactique de la traduction. Mérites de l'analyse conversationnelle face aux TAPs (Thinking Aloud Protocols).

Dans la plupart des cours de traduction dispensés dans le cadre de la formation universitaire des étudiants de langues vivantes règne une sorte de malaise. Les étudiants sont insécurisés: ils n'ont pas l'impression de faire des progrès. Ils ne retrouvent guère les considérations didactiques susceptibles de fournir les bases nécessaires à la structuration d'un cours, à la progression dans le processus d'apprentissage, à une utilisation ciblée d'exercices variés. On constate un certain vide didactique.

Cette carence didactique est due principalement à l'ignorance complète dans laquelle se trouve l'enseignant chargé des cours de traduction en ce qui concerne les comportements traduisants de ses étudiants. Dans la plupart des cas, ils se trouve, en effet, amené à corriger les «fautes de traduction» au stade du produit fini. Les corrections se réduisent alors à la correction de fautes grammaticales ou lexicales; les 
exigences stylistiques relevant souvent de l'utopie et la prise en considération d'éléments extra-linguistiques - comme le contexte socio-culturel - n'effleurant ni l'étudiant, ni l'enseignant. Quant à corriger les comportements traduisants des étudiants - opération qui devrait constituer la finalité première et principale d'un cours de traduction - elle n'est guère envisageable dans la mesure où le produit fini de la traduction ne donne pas accès à ces comportements.

C'est pourtant directement sur ces comportements qu'il faut agir, si l'on veut améliorer la compétence traduisante des apprenants. Et ceci, dans la mesure du possible en tenant compte du fait que chaque individu est une entité structurale, dans laquelle tout se tient, et où, par exemple, un certain comportement face à l'utilisation du dictionnaire peut renvoyer à une certaine conception de la langue.

Dans un article publié en 1987, Jean Klein, Directeur de l'Institut d'Interprètes de l'université de Mons, avait déploré l'impossibilité d'accéder à ces comportements traduisants en ces termes:

... la démarche intellectuelle qui préside à cette opération [l'opération traduisante] reste obscure. Son analyse ne peut s'effectuer qu'à postériori et le traducteur lui-même n'est pas toujours conscient des choix opérés (Klein 1987:62).

Il n'avait apparemment pas connaissance de la recherche qu'avait entreprise H.P. Krings en 1986 sur l'analyse des TAPs (Thinking Aloud Protocols), qui constituaient le corpus de tout ce que ces informateurs avaient pu «penser à voix haute» au cours de l'opération traduisante qu'il leur avait demandé d'effectuer. Krings n'était certs pas le premier à utiliser les méthodes introspectives pour observer les stratégies utilisées pour la solution de problèmes. Il y a eu avant lui Bühler (1907), Clarapède (1917) le maître à penser de Piaget, et d'autres. Mais Krings avait découvert l'introspection au service de la traductologie.

Les recherches de Krings ont fait l'objet de nombreuses critiques: manque de systématisme, manque de cohérence, impossibilité de distinguer entre ce qui est essentiel et ce qui ne l'est pas, caractère artificiel de la situation, manque d'exhaustivité des données fournies, etc. (cf. Stefanink 1986). Ces critiques n'ont pas empêché Lörscher de publier une étude du même type en 1991.

Les recherches de Lörscher et - un peu moins - celles de Krings avaient une finalité epistémologique: reconstruire les chanons de la pensée qui mène de la prise de conscience d'un problème à sa solution. Le projet était ambitieux et les résultats peu convainquants de ce point de vue. Ce type de recherches pourrait toutefois être d'un grand profit pour une exploitation didactique. Ce qui m'a particulièrement gêné cependant, c'est d'une part leur caractère artificiel, d'autre part le manque de motivation chez l'informateur pour aller jusqu'au bout de sa pensée et d'exprimer celle-ci intégralement.

Si j'ai choisi l'analyse conversationnelle pour une exploitation didactique des données introspectives, c'est que, d'une part, la situation de débat dans laquelle se 
trouvent les deux étudiants chargés d'aboutir à une version commune dans la traduction d'un texte n'est nullement artificielle et que, d'autre part, l'exigence d'une version commune les oblige à sortir de leurs retranchements en ce qui concerne leurs argumentations. Ils «négocient» leur traduction et, ce faisant, livrent inconsciemment leurs idées préconçues, naïves face à l'opération traduisante et aux notions impliquées dans cette opération, comme par exemple les notions de mot, de langue, de texte, etc. Les comparaisons que j'ai effectuées entre les TAPs et l'analyse conversationnelle m'ont appris que les données fournies par ces dernières étaient beaucoup plus riches ${ }^{1}$.

Ainsi l'analyse conversationnelle des dialogues enregistrés en cours de traduction m'a révélé que chacun de mes étudiants avait son comportement propre face au texte à traduire, un comportement généralement assez cohérent pour laisser penser que ces décisions en matière de traduction n'étaient pas l'effet du hasard, mais découlaient d'idées reçues inconscientes de la langue et de l'acte traduisant; idées reçues qui induisaient des «maximes de traduction» dictant la conduite à tenir face aux différents problèmes de traduction: ne pas traduire les noms propres, ne pas traduire par un même mot deux mots différents de'la langue source, maintenir à travers tout un texte la traduction d'un mot une fois utilisée, ne pas dépasser la longueur du texte à traduire de plus de $10 \%$, etc.

Parallèlement à ces maximes de traduction induisant les différentes décisions à prendre au niveau micro-structural des différents problèmes, il y a un comportement plus fondamental rélevant d'une conception théorique sous-jacente et inconsciente qui aura pour manifestation concrète que dans un des corpus que je vais présenter, par exemple, l'une des informatrices tentera toujours de résoudre les problèmes de compréhension d'un mot en se limitant à diverses façons d'analyser ce mot alors que sa partenaire fera toujours référence au contexte pour résoudre le même problème.

Pour donner un exemple des résultats didactiques auxquels peut aboutir l'analyse conversationnelle on examinera dans ce qui suit deux corpus émanant, le premier, de la traduction d'un texte réalisée par deux étudiantes allemandes du français vers l'allemand, le deuxième de la traduction de ce même texte vers l'italien, par une enseignante de FLE, de nationalité française douée d'une maîtrise parfaite de l'italien, asistée d'une Roumaine, qui est venue se joindre à elle après avoir traduit le même texte vers le roumain. Le tout se passe en France dans un stage de formation de professeurs de FLE.

\footnotetext{
'Ceci se trouve confirmé dans notre corpus $\mathrm{n}^{\circ} 2$, ou l'informatrice $\mathrm{nl}$ (it.1) travaille d'abord «single», n'ayant pas de partenaire, pour produire un TAP. Notons aussi que Lörscher qui a basé son travail sur les TAPs semble regretter de ne pas avoir choisi l'analyse conversationnelle, puisqu'il essaye, à grand renfort d'argumentations, qui tiennent de l'acrobatie, de faire passer ces TAPs pour des dialogues: «Nevertheless, it can be assumed that implicitly, in the minds of the subjects, the test leader was the addressee of the utterances produced... To this extent, the texts have an implicitly dialogical character» (Lörscher 1991: 57). Il s'agit d'un recours abusif aux licences concédées dans la conception du concept de «conversationnel» décrites par Gülich 1990: 80, cf. Stefanink 1995.
} 


\section{L'ANALYSE CONVERSATIONNELLE COMME RÉVÉLATEUR D'UN COMPORTEMENT COHÉRENT ET SYSTÉMATIQUE FACE AUX DIFFÉRENTS PROBLÈMES À RÉSOUDRE}

L'intérêt didactique d'une démarche visant à analyser le caractère cohérent et systématique des comportements traduisants des étudiants réside dans la possibilité d'agir sur ces comportements à un niveau général, avec un effect multiplicateur au niveau de la solution des différents problèmes d'ordre micro-structural. Cet aspect cohérent du comportement traduisant se manifeste de façon quasi-caricaturale dans notre corpus allemand, où l'informatrice $\mathrm{n}^{0} 1$ (désormais: all. 1) recherche constamment des solutions à partir du mot isolé du contexte dans lequel il se trouve, alors que l'informatrice $\mathrm{n}^{\circ} 2$ (désormais: all. 2) la renvoie inlassablement au contexte pour lui faire comprendre l'absurdité de ses démarches.

Il va sans dire que les comportements que ces informatrices affichent dans leur façons de traiter le texte n'est que la manifestation d'une attitude plus générale face aux choses de la vie. Connaissant bien l'informatrice à qui le respect de l'autorité du dictionnaire interdit de faire confiance à son intuition, je peux affirmer qu'il correspond tout à fait au respect qu'elle a face à l'autorité, face au «non!» du père lacanien. L'exemple qu'elle choisit pour reactualiser hors contexte «en douce», est assez éloquent à ce sujet: «ganz langsam Mädchen» (= tout doux, doucement ma petite).

\section{TYPES DE STRATÉGIES CENTRÉES SUR LE MOT}

Ne connaissant pas le sens de remaniement all. 1 cherche à le trouver par un procédé de décomposition: re + manier (15), alors que le «peut-être dans ce contexte», que lui retorque sa partenaire, indique que cette dernière recherche à trouver le sens à partir du contexte. Pour illico, all. 1 essaye la décomposition soutenue par une étymologisation: essayant de le faire dériver du latin pour ce qui est de «illi» et se référant à l'anglais(!!!) «co» pour la fin du mot: «et illico, bon, illico, est-ce qu'on ne peut pas... illi, illis... du latin .... et co, ça veut bien dire 'ensemble', 'avec'... et illi, ça veut pas dire 'ceux-là', et co kg ou company» (108-111, pour kg elle pense sans doute à «Kommanditgesellschaft»).

Une autre démarche centrée sur le mot est la réactualisation extra-contextuelle: incapable de déduire les sens du mot à partir de son environnement contextuel, l'apprenant se replonge dans des situations qu'il a vécues ou qu'il peut aisément imaginer, afin de faire sortir par association d'idées le sens du mot en question. Krings (1986) utilise pour cela le terme de situational retrieval (qui se limite toutefois aux situations vécues, pas imaginées). Ainsi, malgré une bonne proposition de traduction donnée par all. 2, all. 1 reactualise la locution en aparté hors contexte pour trouver le sens. Ses associations la portent vers le théatre et elle s'éloigne graduellement de la bonne solution qu'avait suggérée all. 2: «Ah oui, 'à part', ça veut aussi dire sur la 
scène... celui qui souffle... le souffleur... ou bien dans une scène, celui qui est à part...oui, lorsqu'ils ne sont pas là ... leur absence pratiquement... 'in absentia'» (40-44).

Elle utilise la même procédure pour la traduction de en douce: les reverbalisations dans la langue cible - «in Ruhe» (= dans le calme; 54, 56), «in Frieden» (= en paix; 56), «Im Guten» (litt. en bien; 58), «im Weichen» (litt.: en mou) - n'ayant pas abouti à un résultat satisfaisant l'all. 1 "reactualise hors contexte», c'est-à-dire qu'elle essaye de retrouver le sens dans sa mémoire longue par association situationnelle en reverbalisant (faussement) dans la langue source la locution qui lui pose problème: «Doucement s'il te plaît», qu'elle traduit par «ganz langsam Mädchen» (= tout doux - ou doucement - ma petite; 60).

Et elle récidive pour casse-croûte qu'elle semble associer à la situation du camping en imaginant quelqu'un qui dit «Laisse casser la croûte maintenant» (219) et «Maintenant c'est la [sic] casse-croûte!», plaçant ainsi le mot «casse-croûte» dans un contexte stimulant qu'elle traduit par «Jetzt geht's los!», «lasst es uns attackieren!» $(211,212)$, qui n'a évidemment plus grand chose à voir avec la connotation prolétarienne que le mot véhicule dans ce contexte et le mépris pour le nouveau conseil des ministres socialistes que l'auteur veut communiquer au lecteur.

Une autre stratégie centrée sur le mot, et qui se manifeste uniquement chez all. 1, est celle que j'appellerais une association abusive de signifiants et qui conduit à une déduction du sens sur la base d'une ressemblance phonique. Sous les contraintes sémantiques du contexte all.1 trouve d'abord intuitivement le sens du mot barbant et le traduit par «langweilig», puis elle revient sur sa traduction et commence à se laisser porter par la chaîne parlée: «heißt barbant ehm langweilig '...nee' barbare, das sind die Barbaren!» (135). Et, pour se couper totalement du signifiant qui a généré cette traduction, elle reverbalise ce lexème trop proche de l'original, comme si elle craignait que la simplicité de ce raisonnement puisse la faire revenir en arrière: elle glose par le corespondant allemand «die Unzivilisierten». Et, lorsque son interlocutrice, quelque peu effrayée par ces escapades sémantiques époustouflantes, lui rappelle que le mot en question se situe dans un contexte qui n'est guère compatible avec sa proposition, elle continue à s'accrocher au signifiant - malgré ces renvois très précis au contexte qui devraient aider à déterminer la sémantisation - et propose «geschwätzig» sous l'influence du mot «bavard» auquel lui fait apparemment penser «barbant». Dans ce deuxième cas on an afaire à une association abusive de signifiants par dérivation.

Elle victime est une autre fois de cette association abusive de signifiants par dérivation lorsqu'elle associe spontanément «casse-croûte» à «Sauerkraut» (291), sous l'influence du mot "choucroûte» que lui rappelle le signifiant de «casse-croûte». (Notons que cette association ne se produit que dans la tête des informateurs allemands!).

Ceci se reproduit pour le mot repérer qu'elle traduit d'abord par «ersetzen» ou «wiederbekommen» (104), sous l'influence analogique du signifiant de «récupéren», pour ensuite se jeter de nouveau à corps perdu dans un délire sémantique, où elle crée le substantif «repération», pour remonter de là à «Kriegsreparation», se pressant 
d'expliquer qu'il s'agit des «dettes de guerre que toutes ces puissances avaient à rembourser après la guerre» (106) afin de rendre sa proposition plus plausible. Un artifice rhétorique, face auquel son interlocutrice semble impuissante, malgré l'absurdité de la proposition. Résignée, de guerre lasse, elle «répond ich hab keine Ahnung» (109), pour finalement l'inviter (avec un soupir) à continuer: «(seufzend) weiter» (122), le «weiter» indiquant que la suite du contexte pourrait lui ouvrir une issue de secours pour échapper à cet enchaînement délirant.

Le fait que l'interlocutrice résigne face à cet emballement rhétorique qui veut forcer sa conviction plutôt que convaincre, et derrière lequel se cache un manque d'assurance plus profond, est plutôt inquiétant. Elle a la même réponse «keine Ahnung» (137), face au même «raisonnement» de all. 1 à propos de «barbants». Ceci ne montre-t-il pas à quel point nous avons affaire à des comportements invétérés et bien assis et qu'all.1 signale clairement un refus de toute discussion à sa partenaire? C'est là que doit intervenir le didacticien pour confronter le patient avec sa logorrhée, provoquer une catharsis et le rendre disponible pour un traitement adéquat, c'est à dire une introductions aux fondements théoriques d'une traductologie!

Cette impression qu'il s'agit là de convictions bien invétérées, dont les protagonistes ne démordent pas aisément, est corroborée par un discours métatraductionnel auquel notre corpus nous offre la chance d'assister et qui présente lui aussi les artifices rhétoriques destinés à masquer l'absence d'un raisonnement. En effet, une troisième informatrice (désormais: all. 3) vient se joindre aux deux premières. Etant venue en retard elle est venue me voir pour me demander ce que les autres faisaient et à quoi cela servait. J'avais mentionné, entre autres, que cela pouvait servir à voir comment les étudiantes utilisaient les dictionnaires. Aussi, lorsqu'elle vient se joindre à nos deux informatrices, qui étaient juste en train de reprendre une deuxième fois leur traduction, après le premier jet auquel nous venons d'assister, elle a dans sa tête la réponse que je lui ai donné sur l'utilisation des dictionnaires. Comme nous l'apprenons au cours de cette conversation, ces deux informatrices s'étaient défendues d'utiliser le dictionnaire. Voyant qu'elles ne s'en sortaient pas, all. 3 leur demande pourquoi elles n'ont pas recours au dictionnaire qui se trouve sur la table, devant eux. Il est frappant de constater à quel point leurs réponses viennent expliciter avec clarté les démarches qu'elles ont suivies tout au long de leur opération traduisante, venant ainsi corroborer les résultats de notre analyse. All. 2 répond que souvent le contexte peut apporter des solutions: «außerdem ergibt sich ja häufig auch was wenn du weiter liest» (233), alors que all. 1 est convaincue que si elles prenaient le dictionnaire, elles trouveraient immédiatement et auraient terminé en deux secondes: «wir wollten nicht im ersten approche sozusagen... Hilfe! dictionary! Deutsches Wort und das ist es, dann könnten wir uns das gleich hier sparen, dann wären wir in zwei Sekundenhier fertig" (240-244) et quand all. 3 nie énergiquement que les choses sont aussi simples, all. 1 est tellement convaincue de sa conception de l'opération traduisante et du rôle que doit y jouer le dictionnaire qu'elle s'obstine en tapant du pied: «ja aber dann trotzdem_rein theoretisch würden wir dann ja finden», le «theoretisch» ne voulant 
évidemment pas dire qu'elle se base sur une théorie, mais ressortant d'un rhétorique qui indique, au contraire, que la «maxime de traduction» irréfléchie à laquelle elle obéit inconsciemment est tellement invétérée qu'elle refuse toute discussion.

\section{L'ANALYSE CONVERSATIONNELLE COMME INSTRUMENT DE MOTIVATION}

Ce refus de discussion est d'autant plus regrettable qu'il s'agit d'un problème fondamental, comme le constate le lexicologue J.-L. Descamps:

Cependant la conscience naïve ... tient plutôt que chaque mot possède (un ou plusieurs) sens en soi: soit un sens plutôt incarné dans une manière de définition ou dans un lot de synonymes approchés, quand il s'agit de la langue maternelle, soit un sens incarné dans une ou plusieurs traductions, quand il s'agit d'une langue étrangère en cours d'apprentissage. (1993: 22)

C'est là que doit intervenir une didactique de la traduction! Lorsque cette informatrice, all.1, tellement réfractaire à toute remise en question de ces idées arrêtées sur l'opération traduisante et, notamment, sur le rôle que doit y jouer le dictionnaire, a été confrontée avec ses propres démarches au cours de la transcription de cette conversation et de l'analyse qui s'en est suivie, elle a eu un choc provocateur d'une catharsis qui l'a sensibilisée pour une autre approche de l'opération traduisante. Il s'agit d'une étudiante qui, le semestre précédant, m'avait demandé si elle pouvait me rendre des copies qu'elle me demandait de corriger sans qu'elle assiste au cours. Elle n'a guère progressé pendant ce semestre. Après cette expérience elle était motivée pour discuter de l'utilisation du dictionnaire, de la nature du mot, de la notion de texte etc. Il y a eu une prise de conscience qui lui a permis de développer ce que Krings a appelé «übersetzerisches Bewußtsein».

\section{L'ANALYSE CONVERSATIONNELLE RÉVÉLATRICE DU RÔLE DE L'INTUITION DANS L'OPÉRATION TRADUISANTE}

L'intérêt didactique d'une telle prise de conscience est essentiel à un niveau plus général de nature épistémologique. Les publications de ces trente dernières années consacrées à l'opération traduisante s'évertuent à développer des modèles d'analyse du texte de plus en plus sophistiqués dont le plus complexe (Gerzymisch-Arbogast, Murdersbach 1998), qui vient de voir le jour dans une collection qui se veut introductrice à des domaines scientifiques réussira certainement à susciter chez le praticien de la traduction, l'attitude de refus face à la théorie, décrite par Hönig, Kußmaul (1982: 9). Le professionnel de la traduction dira qu'il n'a pas besoin de ce fatras théorique, que, lui, il traduit intuitivement. 
Et il n'a pas tout à fait tort. L'analyse conversationnelle revèle que l'intuition joue un rôle bien plus important que celui que veut lui attribuer par ex. Wilss (1992:42), qui accorde une chance à l'intuition seulement après l'échec des autres stratégies pour arriver à une solution:

Da wo sich das sorgfältig aufgebaute Reflexionsgerust der methodisch fundierten Übersetzungsprozeduren als nicht mehr tragfähig erweist, wo Frames, Skripts, Szenarios, Schemata, routinisierte Praktiken, Durchschnittsverhalten versagen, liegt die Chance der Übersetzungsintuition.

Lorsque j'ai mis all.1 en face du discours qu'elle tient à propos de la traduction de barbants, elle l'a trouvé grotesque. Nous sommes convenus qu'il était digne de celui que Sganarelle tient face à Dom Juan chez Molière. Seulement nous ne sommes pas chez Molière, mais nous cherchons à améliorer les stratégies traduisantes. Le manque de confiance de notre informatrice en son intuition exigeait une thérapie qui puisse le donner la confiance nécessaire en son intuition. Pour cela il fallait d'abord attirer l'attention de notre informatrice sur le fait que sa première traduction intuitive par «langweilig» était bonne. Il fallait ensuite lui montrer que cela se répétait pour le mot «pile» (292), qui lui était venu intutitivement pour reverbaliser «pétantes», qu'elle ne connaissait pas. Il fallait lui faire comprendre qu'il y avait des raisons à cela, que «nihil ex nihilo», que c'étaient les contraintes sémantiques du contexte qui avaient induites ces solutions, que si elle a pu comprendre «barbants» comme «langweilig», c'était sous la pression sémantique de «distraire» qui activait les sèmes antonymiques du «joker», sémantique qu'était pour elle le mot «barbants», et que c'était la pression sémantique de l'entourage de «pétantes» («12: 30», «prolongations», «heures») qui l'a induite à sémantiser «pétantes» comme indiquant un précision du point de vue de l'horaire. A travers le choc catharsique provoqué par la confrontation avec sa traduction de «barbant», notre étudiante ressent la motivation nécessaire pour passer à une analyse du texte, qui lui fera comprendre le processus sémantique qui a conduit à son intuition.

L'intuition ainsi conçue, que je qualifierais d' «intuition raisonnée» devient un élément de saisie du sens, qui peut être validé par une analyse subséquente, destinée établir cette «intersubjective Überprüfbarkeit» dont parle Gerzymisch-Arbogast (1994), terme que j'adoucirais en «intersubjective Nachvollziehbarkeit» (Stefanink 1997: 165).

D'une façon générale l'analyse conversationnelle confirme l'antériorité de la saisie intuitive du sens par rapport à l'analyse du texte et inverse ainsi l'ordre établi par les défenseurs de «l'analyse-du-texte-pertinente-pour-le-traducteur» («übersetzerrelevante Textanalyse»), comme par ex. Wilss 1992, Hönig 1989, Nord 1991, Kotcheva 1992, etc. pour lesquels prévaut une interdiction absolue de traduire le moindre mot avant d'avoir fait l'analyse exhaustive du texte (pour plus de détails cf. Stefanink 1997). 


\section{L'ANALYSE CONVERSATIONNELLE FOURNIT AU DIDACTICIEN UN INVENTAIRE DES BESOINS CENTRÉE SUR LE GROUPE CIBLE}

Les questions touchées au cours de l'analyse de notre premier corpus nous ont déjà fourni un certain nombre d'éléments à traiter pour améliorer les comportements traduisants des étudiants, comme les notions de langue et de parole, l'utilisation des dictionnaires, le mot dans le dictionnaire et le mot dans le texte, etc.

Le deuxième corpus, fourni par une traduction du même texte vers l'italien et enregistré dans le cadre d'un stage de formation continue ayant pour thème la traduction et s'adressant à des professeurs de FLE nous révèle ces mêmes besoins d'information concernant les éléments de base impliqués dans l'opération traduisante, comme les notions de langue, de texte etc.

Comme il s'agit de personnes plus âgées, bien assises dans la vie professionnelle d'enseignants, ayant déjà eu à faire des traductions dont elles devaient assumer la responsablité dans une interaction sociale et non pas dans la tour d'ivoire universitaire on peut sentir chez elles une prise en considération de facteurs auxquels les étudiantes n'avaient pas pensé.

Notons d'abord que nous retrouvons la même dichotomie dans les attitudes fondamentales par rapport à la traduction (même si dans ce court corpus elles ne sont pas aussi marquées et sans que cela implique que cette dichotomie soit une règle générale, mais sans doute l'âpreté avec laquelle se déroule ce processus de «négociation» contribue-t-elle à faire ressortir les traits caractéristiques de chacun des négociateurs. Ainsi lorsque l'informatrice italianisante (désormais: it. 1) dit à propos d'une phrase que la Roumaine (désormais: it. 2) qualifie de «très moche» (481):

j'avais sauté tranquille, moi, je saute quand ça va pas, parce que j'aime avoir tout le texte, puis, après, reprendre en plusieurs fois (482-484).

(un comportement traduisant concentrique, qui caractérise d'ailleurs le traducteur professionnel face à la démarche linéaire du non-professionnel, selon Krings 1987) - elle manifeste un comportement contextuel face à it. 2 qui trahit à diverses reprises un attachement au mot comme nous le verrons dans les exemples qui suivent.

De même nous retrouvons, comme dans notre premier corpus une méconnaissance totale quant à la notion de texte. Lorsque it. 2 dit:

tu ne peux pas être fidèle au texte et en même temps toucher la satire,

c'est ce qui me gêne (614-615)

elle implique que, pour elle, le mot «texte» signifie la lettre du texte, la suite de mots pris individuellement, et non pas le sens qui s'en dégage et qui est le vrai message du texte, message dont elle a pourtant conscience, puisqu'elle parle de la satire. Ce qui est frappant, c'est que sa partenaire, qui a une démarche traduisante intuitivement meilleure, commet la même erreur de raisonnement quand elle essaye de donner une justification théorique, en argumentant:

moi je suis obligée de m'éloigner du texte... on rend plus l'esprit de ce texte

qui est ironique (609-610) 
On le voit, même cette traductrice professionnelle, plus expérimentée a des besoins d'information théorique pour justifier sa démarche, sinon elle restera toujours mal à l'aise dans sa peau, avec le sentiment bien connu du «traduttore - traditore», «traduction - trahison!». Ce n'est qu'à travers un fondement théorique de la notion de texte comme nous le donne par ex. Stolze 1992 avec son concept de la «Übersummativität des Textes» que l'on arrivera à donner à cette traductrice l'assurance nécessaire face au récepteur de la traduction, qui n'est pas un professionnel et qui est souvent deçu jusqu'à devenir agressif, lorsqu'il ne retrouve pas dans le texte cible le mot que lui donne le dictionnaire bilingue ou sa connaissance rudimentaire de la langue cible (cf. Hönig 1995: 22-24). Le didacticien essayera d'approfondir dans une réflexion commune avec l'informatrice, ce qu'impliquait pour elle cette notion d'«esprit».

De même, la réflexion sur la nature des langues par laquelle it. 2 cherche à expliquer ses défaillances traduisantes:

il y a des langues qui sont plutôt sèches, raides (624)

et dans laquelle le «plutôt» trahit déjà un manque d'assurance par rapport à ce qu'elle dit, pourait donner lieu à un approfondissement de la notion de langue. Ainsi l'article d' A. Martinet (1965), intitulé «Une langue peut-elle être belle?» pourrait susciter une discussion enrichissante à ce sujet.

D'une façon générale on peut noter chez ces deux traductrices avec une expérience professionnelle chez it. 1 et semi-professionnelle chez it. 2 une plus grande prise en considération du public cible et de son back ground socio-culturel:

et si tu regardes les journaux italiens par exemple (541).

Comparées aux étudiantes du corpus allemand, elles assument leurs responsabilités, elles veulent une traduction communicative:

mais c'est à toi, traductrice, de rendre le texte intelligible aux gens auxquels tu t'adresses (530-531).

C'est à partir de ces remarques que l'enseignant peut sensibiliser les étudiants à une approche théorique en leur montrant que leurs remarques sont pertinentes et se situent dans un cadre théorique précis.

Là ou elles ne sont plus d'accord, c'est sur la façon de compenser les pertes d'information pour le récepteur en LC. It. 2, attachée à la traduction des mots reconnaît, certes, «il faut que tu précises, peut-être» (ou le «peut-être» revèle une fois de plus, un manque d'assurance qui ne pourra être comblé que par une réflexion théorique),

mais pas à l'intérieur du texte, parce que tu n'as pas le droit de reécrire le texte (524-525)

c'est légalement interdit (528)

se référant à une maxime de légalité, dénuée de tout fondement.

Et on arrive à l'éternel débat qui préoccupe tout traducteur: faut il mettre des annotations, des parenthèses, une introduction ou une traduction explicative? It. 1 argumente

moi je le mets entre parenthèses, si tu regardes les journaux italiens, par exemple, à propos d'un ministre à l'étranger, par exemple, ils te mettent entre parenthèses (540-545) 
Masi elle n'arrive pas à convaincre son interlocutrice qui lui répond:

mais ce sont eux-mêmes qui le font, c'est pas à l'intérieur d'une traduction (547-548)

si tu écris l'article toi-même, d'accord, à ce moment-là tu précises, mais si tu traduis, je ne sais pas si tu peu le faire, ou, alors, tu le fais dans une introduction (556-559)

Ce à quoi it. 1 répond:

ou tu fais des notes (560)

Mais it. 2, trouvant cela sans doute trop près du «texte» relance le débat en proposant de rejeter les notes à la fin:

des notes à donner à la fin (561)

It. 1 nuance en répondant que cela va pour un livre, mais pas pour un article de journal:

dans un livre, justement, ou tu peux te référer à des pages et tout, mais dans un article de journal, c'est pas possible (562-565)

Là encore nous assistons à un débat qui s'éternise parce qu'il n'est pas porté par une théorie. Il faut profiter de leur intérêt pour la question en leur faisant comprendre qu'il s'agit d'un débat fondamențal. Une réflexion sur la typologie des textes pertinente pour le traducteur, ainsi que sur la finalité de la traduction serait d'un grand secours pour les prises de décision à ce niveau micro-structural. Je pense aux trois types de texte proposé dans Reiß 1972 et à la Skopostheorie élaborée par Reiß, Vermeer 1984. La lecture de Lederer 1994 lui apprendrait aussi qu'en donnant une traduction explicative, elle ne «rajoute» pas quelque chose au texte, elle ne «change» pas le texte et elle ne «reécrit» pas le texte, comme elle prétend à pluseiurs reprises, mais elle «explicite», ce qui est implicite pour le lecteur en raison de son back-ground socio-culturel à lui, et qui doit être explicité pour le lecteur en LC si l'on veut maintenir la Wirkungsgleichheit préconisée par Reiß, Vermeer.

Cette typologie des textes associée à la Skopostheorie fournira aussi des critères de décision quant à l'autre problème de traduction qui se pose à it.2: a-t-on le droit d'introduire des anglicismes? Face à la traduction de «casse-croûte» par «snack», par it.1, elle a un sursaut:

Oh, non c'est un anglicisme (737)

L'argumentation avec laquelle it. 1 défend sa traduction n'est évidemment pas convainquante, parce que sans fondement théorique:

tu ne peux pas trouver un journal italien sans un article où tu as cinquantemille anglicismes (737-740)

Et pourtant it. 2 sent très bien que quelque chose ne va pas avec ce mot de «cassecroûte». D'abord elle dit que la traduction par «snack» «diminue le repas» (731), puis elle argumente que c'est un anglicisme. En réalité elle a le sentiment juste que le mot est utilisé ici à contre-courant, qu'il est inattendu:

ce n'est pas casse-croûte à midi pétantes, ce n'est pas du tout casse-croûte! (729-730). 
La confrontation avec le cheminement de sa propre pensée la motivera à se familiariser avec une théorie de la traduction qui lui apprendrait que dans ce texte tous ces efforts pour traduire la nature précise du contenu de ce repas sont vains, que le choix du mot «casse-croûte» par l'auteur, qui exclut d'autres choix paradigmatiques, est conscient et a pour fonction de soutenir la fonction globale du texte dans lequel l'auteur présente le nouveau conseil des ministres socialistes comme composé de prolétaires n'ayant aucun sens des responsablités. Cette analyse conversationnelle la motivera aussi pour une analyse du texte, qui lui montrera comment le contexte immédiat de «casse-croûte» vient soutenir cette lecture (connotations prolétariennes de «plus question de jouer les prolongations», de «pétantes»), que ce texte n'est pas un texte «informatif» qui veut nous renseigner sur ce que les ministres mangent à 12:30 heures, mais que le rhème de ce texte est le manque de responsabilité des ministres et que c'est ce rhème qu'il faut «traduire!» (cf. Stefanink 1997).

Finalement la pertinence de la Skopostheorie est corroborée par la remarque de it. 1, qui, devant tous ces problèmes qui se posent, fait remarquer à juste titre que

l'exercice en soi est un peu artificiel (567)

ce qui signale à l'enseignant qu'il ne peut pas simplement donner des textes à traduire sans indiquer le contexte dans lequel se situe le texte, la fonction de la traduction et le public auquel elle s'adresse (cf. La Skopostheorie de K. Reiß). Ceci nous amène à parler des répercussions que l'analyse conversationnelle doit avoir sur la didactique de la traduction.

N.B.: Il est vrai que ces problèmes qui ont suscité son argumentation ne se posaient pas de façon aussi aiguë tant qu'elle travaillait seule en pensant à voix haute, comme le montrent les 15 pages de corpus qui précèdent. Ce sont les idées opposées de sa partenaire en traduction qui ont suscité cette extériorisation de ce qui était implicite et inconscient dans sa démarche.

\section{CONCLUSIONS POUR UNE DIDACTIQUE DE LA TRADUCTION}

Quelles conclusions tirer de ces analyses conversationnelles?

Au niveau microstructural du texte, elles nous permettent de procéder à une évaluation des stratégies utilisées par les apprenants. Et ceci non seulement pour condamner des erreurs aussi évidentes que la recherche de solutions centrées exclusivement sur le mot, mais aussi pour corriger certaines idées préconçues défendues même par des spécialistes. Ainsi les résultats obtenus par all. 1 - qui fait partie de ses stratégies de base puisqu'elle y reviendra plus tard:

casser la croûte heißt so, wenn man anfange will zu essen, dann sagt man eh wenn man alles präpariert hat, schon den Tisch gedeckt hat und dann setzt man sich hin und sagt man: bon, maintenant on va casser la croûte, jetzt geht's ran an den poulet und dann mit dieser Hände-, Händebewegung, so ungefähr kann ich mich noch erinnem (1077-1084) 
montrent clairement que le situational retrieval pour trouver le sens d'un mot est à condamner, malgré les regrets de Krings (1986: 340, 511, 512), qui déplore le fait que les étudiants n'aient pas assez souvent recours à cette stratégie.

Souvent - surtout chez les apprenants qui ont déjà une certaine expérience de la tradcution, comme les informatrices de notre second corpus - les réflexions naïves émises au cours de la négociation de la traduction fournissent à l'enseignant une base pour situer ces réflexions dans un cadre théorique plus solide, plus élaboré.

Au niveau de la structuration du cours, l'analyse conversationnelle menée au début d'un cours de traduction permet d'une part à l'enseignant de présenter aux étudiants leurs propres déficits au plan théorique et de créer ainsi la motivation nécessaire pour accepter de se familiariser avec les éléments de théorie susceptibles de combler ces déficits et d'améliorer la compétence traduisante. Elle lui fournit, d'autre par, une base pour l'analyse des besoins, nécessaire pour la structuration d'un cours qui se voudra centré sur les besoins des apprenants du groupe cible face auquel il se trouve. Nous avons vu que ces besoins peuvent être accentués différemment d'un groupe à l'autre.

Au niveau de la structuration du cursus des langues vivantes à l'université, l'analyse conversationnelle rend manifeste le besoin d'une revalorisation du cours de traduction dans le cadre du cursus. En Allemagne, enseignants et étudiants sont victimes de l'institution universitaire qui situe le cours de traduction le domaine de la «Sprachpraxis» et qu'il consiste dans des «exercices de traduction» («Übersetzen üben»). L'étiquette de «Sprachpraxis», impliquant que l'étudiant ne pourra pas écrire un des 4 ou 5 mémoires de nature théorique («Hausarbeit») qu'il doit remettre au cours des ses études ${ }^{2}$. Comment veut-on alors que l'étudiant ne soit pas convaincu que pour apprendre à traduire il faut traduire, «traduire et encore une fois traduire» (attitude critiquée par Hönig, Kußmaul 1982: 9). L' «exercice» de traduction fait pour ainsi dire partie des «basses besognes» qu'on laisse exécuter par le lecteur de langue étrangère qui vient faire deux ou quatre années dans le pays de la langue qu'il étudie pour y rédiger sa thèse de doctorat, mais qui, évidemment n'a jamais eu une formation dans cette discipline. En France, la situation ne semble guère meilleure, puisque, au cours de mon lectorat dans une université française on me demandait de tester chaque semaine l'apprentissage de 5 pages de vocabulaire, classé par ordre alphabétique (!) au début de chaque cours de traduction. Ceci avant la traduction du texte hebdomadaire (!), qui était de rigueur. Comment peut-on alors s'étonner que les étudiants «collent au mot»? Lorsque je retrouvais un des mots de la liste dans des contextes de traductions où il ne «collait» pas du tout, les étudiants semblaient ne pas comprendre que je n'apprécie pas, «puisque c'était comme ça dans la liste» et qu'ils pouvaient me montrer qu'ils avaient bien appris leur liste.

\footnotetext{
${ }^{2}$ Lorsque, il y quelques années on a procédé à l'université de Bielefeld à la catégorisation des cours par domaines («Bereiche»), chaque enseigant se défendait contre un classement de ses cours dans la catégorie «Sprachpraxis». Comme on se demandait ce que finalement on allait mettre dans la Sprachpraxis, un linguiste germaniste s'est écrié: «Mais la traduction, ça, pour sûr, c’est de la Sprachpraxis!», à la satisfaction quasi-unanime de ses collègues.
} 
Face à cette situation et me basant sur les leçons que j'ai pu tirer des analyses conversationnelles de mes étudiants, je préconise un cours d'introduction à la traduction, qui commencera par une expérience de traduction par groupes de deux ou trois enregistrée et transcrite par les étudiants, lors de la première séance, et qui a pour but de les rendre réceptifs à une action sur leurs comportements traduisants, en leur montrant que leurs esprits ne sont pas vierges d'idées préconçues sur la traduction et qu'ils ont intérêt à faire le ménage dans leurs têtes avant de passer à l'acte traduisant. Les étudiants procèdent ensuite, avec l'aide de l'enseignant, à l'analyse de leurs propres comportements traduisants et l'on établit en commun une liste des besoins à combler.

Ce cours sera la condition pour accéder au cours de préparation à l'examen final, dans lequel les étudiants seront préparés à traduire deux ou trois types de texte parmi lesquels ils pourront choisir le jour de l'examen final. Ceci leur fournit une motivation supplémentaire pour s'enîtraner, puisqu'il connaîtront le type de texte qui servira de test de connaissances. Ce test peut ainsi juger des connaissances acquises «achievement testing» (Hatim, Mason 1998: 200) qui est à préférer à un examen où les étudiants s'attendent à ce «que le ciel leur tombe sur la tête», comme cela a été le cas pour une de nos étudiantes françaises, qui, après un séjour d'une année en Allemagne avait une excellente maitrise de la langue de Goethe, mais qui s'est vu éliminée avec une note catastrophique pour ne pas avoir su traduire un texte portant sur le foot-ball. Apparemment le correcteur avait critiqué son manque de vocabulaire technique dans ce domaine. On est moins sévère dans la pratique professionnelle où le traducteur doit à chaque fois s'initier au domaine de spécialité d'où provient le texte à traduire.

L'analyse conversationnelle déterminera donc aussi les modalités de l'examen final, du test de connaissances acquises, qui devra clairement formuler la demande du donneur d'ordre qu'est l'enseignant par rapport au traducteur qu'est l'étudiant. Elle déterminera également le choix des textes à traiter au cours et à traduire à l'examen.

Ces deux exemples de corpus très courts nous ont déjà montré la richesse des enseignements que peut tirer un didacticien de la traduction qui est prêt à s'intéresser «à l'ordre des choses dans la tête des gens» (Gülich 1990: 73) comme le préconise l'ethnoscience et qui veut étudier le «savoir commun et quotidien dont disposent» ses étudiants concernant la traduction. Appelons Ethnotraductologie la science qui s'intéresse à ces connaissances!

\section{OUVRAGES DE RÉFÉRENCE}

Arntz, R. (Hrsg.) (1987), Textlinguistik und Fachsprache, Hildesheim: Olms.

Desca mps, J.-L. (1993), Dictionnaire contextuel de la langue politique, Paris: Didier.

Gerzy mi sch-Arbog ast, H. (1994), Übersetzungswissenschaftliches Propädeutikum. Grundlagen zu einer wissenschaftlichen Methodik des Übersetzens. Tübingen: Francke.

Gerzymisch-Arbogast, H., Murdersbach, K. (1998), Methoden des wissenschaftlichen Übersetzens, Tübingen: Francke. 
Gülich, E. (1990), Pour une ethnométhodologie linguistique. Description des séquences conversationnelles explicatives, in: Charolles, M., Fischer, S., Javez, J. (éds.), Le discours, représentations et interprétations, Nancy, 60-70.

Hati m, B., Mas on, I. (1997), The Translator as Communicator, London: Routledge.

Hönig, H. G. (1989), Die übersetzerrelevante Textanalyse, in: Königs.

Höni g, H. G., Ku Bmau l, P. (1982), Strategie der Übersetzung. Ein Lehr- und Arbeitsbuch, Tübingen: Narr.

König s, F. G. (Hrgs.) (1989), Übersetzungswissenschaft und Fremdsprachenunterricht. Neue Beiträge zu einem alten Thema. München: Goethe-Institut.

Kotcheva, K. (1992), Probleme des literarischen Übersetzens aus textlinguistischer Sicht, dargestellt am Beispiel bulgarischer Übersetzungen zu Prosatexten aus der deutschen Gegenwartsliteratur, Frankfurt/M: Peter Lang.

Kring s, H. P. (1986), Was in den Köpfen von Übersetzern vorgeht. Eine empirische Untersuchung zur Struktur des Übersetzungsprozesses an fortgeschrittenen Französischlernem. Tübingen, Narr.

Krings, H. P. (1987), Blick in die 'Black Box' - Eine Fallstudie zum Übersetzungsprozeß bei Berufsübersetzerm, in: Arntz.

Lederer, M. (1994), La traduction aujourd'hui, Paris: Nathan.

Lörscher, W. (1991), Translation Performance, Translation Process, and Translation Strategies, Tübingen: Narr.

Reiss, K. (1971), Möglichkeiten und Grenzen der Übersetzungskritik, München: Hueber.

Re is s, K., Vermeer, H. J. (1984), Grundlegung einer allgemeinen Translationstheorie, Tübingen: Niemeyer.

Ste fanink, B. (1986), Review Article zu Krings 1986, Bielefelder Beiträge zur Sprachlehrforschung $15,169-186$.

Stefanink, B. (1988), Über Stellenwert und Inhalt von Übersetzungskursen im universitären Fremdsprachenunterricht, Fremdsprachen lehren und lernen 17, 194-209.

Stefanink, B. (1991), Vom Nutzen und der Notwendigkeit der Theorie für den universitären Übersetzungsunterricht, in: The Role of Translation in Foreign Language Teaching, TRIANGLE 10, Paris: Didier Erudition, 59-84.

Stefanink, B. (1993a), Traduire: De la théorie à la pratique, Le français dans le monde, Paris, janv. 1993.

Stef a n ink, B. (1993b), Übersetzen als fünfte Fertigkeit, Zielsprache Französisch, 1993/2.

Stefanink, B. (1995a), Le traducteur et les mots, Le français dans le monde, $n^{\circ} 275$, (août-sept.) 1995: 38-43.

Ste fa n ink, B. (1995b), L'ethnotraductologie au service d'un enseignement de la traduction centré sur l'apprenant, Le langage et l'homme, 1995, nº 4 (octobre) 265-293.

Stefanink, B. (1995c), Review Article zu Lörscher 1991, Fremdsprachen Lehren und Lermen, Tübingen: Narr, 1995, 271-278.

Stef a n ink, B. (1996), Théories et enseignement de la traduction, Le journal du traducteur, Paris, janv. $2-15$.

Stef an ink, B. (1997), 'Esprit de finesse' - 'Esprit de géométrie': Das Verhältnis von 'Intuition' und 'übersetzerrelevanter Textanalyse' beim Übersetzen, in: Rudi Keller (Hrsg.), Linguistik und Literaturübersetzen, Tübingen: Narr, 161-183.

Stolze, R. (1994), Übersetzungstheorien. Eine Einführung, Tübingen: Narr.

Wilss, W. (1992), Übersetzungsfertigkeit. Annäherung an einen komplexen übersetzungspraktischen Begriff, Tübingen: Narr. 


\section{ANNEXE \\ Le mercredi, à l'Elysée \\ Réunion du conseil de discipline}

Avant le dernier remaniement, ils étaient trente-six (en comptant Mitterrand) autour de la table du Conseil des ministres. Ils ne sont plus aujourd'hui que dix-sept. La table étant restée la même, ces messieurs-dames ont pu se desserrer.

Mais à entendre certains d'entre eux, cette aération comporte des inconvénients. Les discussions en aparté sont désormais à peu près impossibles et, lorsqu'un ministre intervient, il devient très risqué d'échanger en douce quelques vacheries à son endroit. L'oeil noir de Tonton fusille immédiatement le bavard.

Alain Savary, dont c'était un des sports favoris jusqu'à cette regrettable petite révolution, est, paraît-il, terriblement frustré.

La classe étant moins nombreuse, et plus facile à surveiller, il est devenu quasiment impossible de lire tranquillement son journal ou de faire son courrier sans se faire repérer illico.

Autre petit jeu pratiquement refusé désormais aux ministres: la rédaction des petits mots que, traditionnellement, ils se passent de l'un à l'autre pour se distraire au cours des exposés parfois barbants de leurs distingués collègues.

Et puis, le brouhaha est proscrit.

Tonton a voulu imprimer un style nouveau au Conseil. Maintenant, il donne systématiquement la parole à tous ceux qui la demandent, et il insiste même pour avoir l'avis des principaux ministres sur tel ou tel sujet. Ce qui fournit, paraît-il, l'occasion à Michel Rocard de faire de véritables exposés dans le style Sciences-Po.

Enfin, le Conseil doit être désormais terminé à 12 h 30 pétantes. Plus question de jouer les prolongations. L'heure du casse-croûte, c'est sacré. 